\title{
Effects of 5-mg dose of olanzapine for breakthrough nausea and vomiting in patients receiving carboplatin-based chemotherapy: a prospective trial
}

\begin{abstract}
Akimitsu Maeda ${ }^{1} \wedge$, Hiroki Yoshida ${ }^{2} \wedge$, Hirotaka Inoue ${ }^{3}$, Masayuki Ejiri $^{4}$, Satoe Yamaguchi ${ }^{5}$, Hideyuki Kushihara $^{6}$, Yoshihiro Yamamoto ${ }^{7}$, Yosuke Ando $^{8}$, Yumiko Sato ${ }^{9}$, Yuusuke Tashiro ${ }^{10}$, Ayako Hasegawa ${ }^{1}$, Yuko Takahara ${ }^{2}$, Mika Mizutani ${ }^{3}$, Isao Oze ${ }^{11}$, Junichi Shimizu ${ }^{12}$

${ }^{1}$ Department of Pharmacy, Aichi Cancer Center Hospital, Nagoya, Japan; ${ }^{2}$ Department of Pharmacy, Japanese Red Cross Nagoya Daini Hospital, Nagoya, Japan; ${ }^{3}$ Department of Pharmacy, National Hospital Organization Nagoya Medical Center, Nagoya, Japan; ${ }^{4}$ Department of Pharmacy, Aichi Medical University, Nagakute, Japan; ${ }^{5}$ Department of Pharmacy, Chubu Rosai Hospital, Minato-ku Nagoya, Japan; ${ }^{6}$ Department of Pharmacy, Japanese Red Cross Nagoya Daiichi Hospital, Nagoya, Japan; ${ }^{7}$ Department of Pharmacy, Komaki City Hospital, Komaki, Japan; ${ }^{8}$ Department of Clinical Pharmacy, Fujita Health University School of Medicine, Toyoake, Japan; ${ }^{9}$ Department of Pharmacy, Nagoya City West Medical Center, Nagoya, Japan; ${ }^{10}$ Department of Pharmacy, Nagoya City University Hospital, Nagoya, Japan; ${ }^{11}$ Division of Cancer Epidemiology and Prevention, Aichi Cancer Center Research Institute, Nagoya, Japan; ${ }^{12}$ Department of Thoracic Oncology, Aichi Cancer Center Hospital, Nagoya, Japan

Contributions: (I) Conception and design: All authors; (II) Administrative support: All authors; (III) Provision of study materials or patients: A Maeda, H Yoshida, H Inoue, M Ejiri, S Yamaguchi, H Kushihara, Y Yamamoto, Y Ando, Y Sato, Y Tashiro, A Hasegawa, Y Takahara, M Mizutani, J Shimizu; (IV) Collection and assembly of data: A Maeda, A Hasegawa, I Oze; (V) Data analysis and interpretation: All authors; (VI) Manuscript writing: All authors; (VII) Final approval of manuscript: All authors.

Correspondence to: Akimitsu Maeda. Department of Pharmacy, Aichi Cancer Center Hospital, 1-1 Kanokoden, Chikusa-ku, Nagoya, Aichi 464-8681, Japan. Email: m.akimitsu@aichi-cc.jp.
\end{abstract}

\begin{abstract}
Background: Olanzapine $10 \mathrm{mg}$ is recommended for breakthrough chemotherapy-induced nausea and vomiting. However, there is a possibility that $5 \mathrm{mg}$ can be expected to be sufficiently effective. We aimed to investigate the efficacy and safety of olanzapine $5 \mathrm{mg}$ for breakthrough chemotherapy-induced nausea and vomiting.

Methods: A single-arm prospective trial of olanzapine $5 \mathrm{mg}$ every $24 \mathrm{~h}$ for $72 \mathrm{~h}$ was conducted to treat breakthrough chemotherapy-induced nausea and vomiting in patients receiving carboplatinbased chemotherapy. The primary endpoint was total control (i.e., no emesis, no nausea, and no rescue medications) over $72 \mathrm{~h}$. The secondary endpoints were early efficacy using the nausea scores at 30, 60, and 120 min after taking olanzapine from baseline and adverse events.
\end{abstract}

Results: Among 84 potentially eligible patients, 19 patients who took olanzapine for breakthrough chemotherapy-induced nausea and vomiting were examined. The total control rate was 32\% (95\% CI: $13-$ $57 \%$ ), 65\% (95\% CI: 38-89\%), 65\% (95\% CI: 38-89\%), and 29\% (95\% CI: 10-56\%) during 2-24, 24-48, 48-72 h, and overall period, respectively. The nausea scale significantly reduced after $30 \mathrm{~min}(\mathrm{P}=0.0078)$, and the scale had been reduced by $67 \%$ from the baseline after $60 \mathrm{~min}$. The adverse event of somnolence of any grade was observed in 13 (68\%) patients, 6 (32\%) of whom had grade 2 and 1 (5\%) grade 3 somnolence.

Conclusions: Olanzapine $5 \mathrm{mg}$ did not show the expected effect on the complete disappearance of breakthrough chemotherapy-induced nausea and vomiting within $24 \mathrm{~h}$.

Keywords: Breakthrough; carboplatin; nausea; olanzapine; vomiting

^ ORCID: Akimitsu Maeda 0000-0002-0709-3911; Hiroki Yoshida 0000-0003-4907-7191; Yoshihiro Yamamoto 0000-0002-1143-5830. 
Submitted Sep 08, 2020. Accepted for publication Dec 07, 2020.

doi: 10.21037/apm-20-1784

View this article at: http://dx.doi.org/10.21037/apm-20-1784

\section{Introduction}

Breakthrough chemotherapy-induced nausea and vomiting (CINV) is defined as nausea and/or vomiting that occurs as a result of chemotherapy despite prophylaxis with appropriate antiemetics. It is treated by adding an antiemetic with a different mechanism of action than those given as prophylaxis. Although metoclopramide and prochlorperazine are often used for this purpose, their antiemetic effect is poor $(1,2)$.

Olanzapine blocks multiple neurotransmitters, including dopamine at the dopamine 2 receptor and serotonin at the 5 -hydroxytryptamine 2 and $3\left(5-\mathrm{HT}_{3}\right)$ receptors, sites involved in the nausea and vomiting pathway. The National Comprehensive Cancer Network (NCCN) and the Multinational Association of Supportive Care in Cancer/European Society for Medical Oncology guidelines recommend olanzapine for breakthrough CINV if it has not been used prophylactically because it is more effective in controlling breakthrough symptoms than is metoclopramide (3-5).

However, a recent recommendation is to use olanzapine prophylactically for patients being treated with highly emetogenic chemotherapy such as cisplatin and anthracycline/ cyclophosphamide regimens (6). Therefore, in patients treated with these chemotherapies, an opportunity to administer olanzapine for breakthrough CINV may be reduced.

In contrast, in patients receiving carboplatin-based chemotherapy, triple combination antiemetic therapy of neurokinin (NK)-1 receptor antagonist, $5-\mathrm{HT}_{3}$ receptor antagonist, and dexamethasone has been demonstrated to portray excellent effects in preventing CINV (7). Therefore, we speculate that the benefit of prophylactic olanzapine in all patients receiving the first cycle of carboplatin-based chemotherapy is poor to avoid the olanzapine's side effects. Instead, it is clinically important to investigate the efficacy of olanzapine for breakthrough CINV in patients receiving carboplatin-based chemotherapy.

In a study by Navari et al. (3), the efficacy of a $10-\mathrm{mg}$ dose of olanzapine for breakthrough CINV after highly emetic chemotherapy (cisplatin, or doxorubicin and cyclophosphamide) was demonstrated. Therefore, the generally recommended dose of olanzapine for breakthrough symptoms is $10 \mathrm{mg} /$ day for 3 days (3). However, in a study of CINV prophylaxis in patients receiving highly emetic chemotherapy, $10 \mathrm{mg}$ of olanzapine was not clearly superior in antiemetic efficacy than $5 \mathrm{mg}$ and was more prone to the adverse event of somnolence (8). The approved starting dose for antiemetic therapy is $5 \mathrm{mg}$ in Japan. We therefore hypothesized that a $5-\mathrm{mg}$ dose of olanzapine might have an adequate antiemetic effect for breakthrough CINV as well, this dosage which has not been investigated for this purpose.

Conventionally, studies that directly compare the efficacy of olanzapine 10 and $5 \mathrm{mg}$ in patients receiving carboplatin-based chemotherapy are most desirable solution to this clinical question. However, with the development of prophylaxis antiemetic therapy, if trying to conduct a multigroup comparative study, the enrolment of subjects with onset of breakthrough CINV requires an enormous number of patient registration. Since the evidence of $5 \mathrm{mg}$ olanzapine for breakthrough CINV was little, we conducted a multicenter, open-label, single-arm preliminary trail to evaluate the efficacy and safety of $5 \mathrm{mg}$ of olanzapine for breakthrough CINV in patients receiving carboplatin-based chemotherapy without prophylactic olanzapine.

We present the following article in accordance with the TREND reporting checklist (available at http://dx.doi. org/10.21037/apm-20-1784).

\section{Methods}

\section{Patient selection}

This study was a single-arm prospective trial conducted at 10 hospitals in Aichi prefecture in Japan from April 2018 to March 2019. Patients recruited for the study were 20 years or older with a malignant disease other than hematologic malignancy and in whom a chemotherapy regimen containing carboplatin (area under the curve (AUC) $\geq 5 \mathrm{mg} / \mathrm{mL} \cdot \mathrm{min}$ ) without the prophylactic use of olanzapine was planned. Patients administered anticancer drugs (including oral drugs) during days 2-7 of the regimen and those who had nausea in the 24 -h period prior to chemotherapy were excluded.

Further inclusion criteria were that patients have a serum bilirubin $<2.0 \mathrm{mg} / \mathrm{dL}$ and aspartate aminotransferase or 
alanine aminotransferase $<5$ times the upper limit of normal, an Eastern Cooperative Oncology Group performance status of $0-2$, that they be capable of normal oral intake, and that they were naive to moderately or highly emetic chemotherapy.

The exclusion criteria were a general condition preventing treatment with antineoplastic agents, symptomatic brain metastasis, severe cognitive compromise, seizure disorders requiring anticonvulsant therapy, and a history of hypersensitivity or allergy to olanzapine or similar compounds. Women who were pregnant or breastfeeding were excluded, as were patients being treated for diabetes or who had undergone radiotherapy (except bone irradiation), those with symptomatic ascites that need therapeutic drainage, or who were scheduled to receive it during the 6 days before or after the start of chemotherapy. We also excluded regular users of antiemetic drugs other than as prescribed for CINV prophylaxis at study entry, such as $5-\mathrm{HT}_{3}$ receptor antagonists, corticosteroids, dopamine antagonists, phenothiazines tranquilizers, antihistamines, and benzodiazepines. In addition, patients who started taking opioids within 1 week of chemotherapy and who had nausea at the start of chemotherapy were excluded.

All patients were Japanese, and written informed consent was obtained from all patients before chemotherapy. This clinical trial was approved by the Ethics Committee of each hospital (No.: 2018-1-282) and was conducted in accordance with the Declaration of Helsinki (as revised in 2013). This study was registered with the University Hospital Medical Information Network Clinical Trials Registry (UMIN-CTR), (Study ID: UMIN000031823).

\section{Procedures and treatment regimen}

Patient data, including age, gender, habitual drinking (defined as drinking an alcoholic beverage more than once a week) were recorded at study entry. Physical evaluations and laboratory tests were performed before initiation of treatment.

All recruited patients received standard prophylactic antiemetic therapy with a $5-\mathrm{HT}_{3}$ receptor antagonist and dexamethasone and/or an $\mathrm{NK}-1$ receptor antagonist without olanzapine at the discretion of the physician. The prophylactic antiemetic drugs were not assigned to examine the effects of olanzapine for breakthrough CINV under different preventive antiemetic therapies. The study patients were those who had breakthrough CINV within the $120 \mathrm{~h}$ after carboplatin administration that was treated with olanzapine. These patients were able to start taking olanzapine $5 \mathrm{mg}$ orally when they felt nausea by themselves. From that point on, they took olanzapine every $24 \mathrm{~h}$ (minimum $22 \mathrm{~h}$ ) for $72 \mathrm{~h}$. Patients were informed at the time of study enrolment that olanzapine could be reduced if intolerant. The tolerability was assessed every $24 \mathrm{~h}$. Dose reductions to $2.5 \mathrm{mg}$ were allowed if the patient had somnolence grade 2 or more and asked for a reduced dose (9).

To assess the early efficacy of the treatment, a numerical response scale of $0-10$, which has been used in some similar studies (9), was investigated using a questionnaire at baseline, 30, 60, and 120 min after taking olanzapine. In order to assess the continuity of antiemetic effect and adverse events of the treatment, the following information in 2-24, 24-48, and 48-72 h after taking olanzapine was collected every $24 \mathrm{~h}$ using a questionnaire: the frequency of vomiting (including gagging), additional use of other rescue medications such as metoclopramide and prochlorperazine for CINV, maximum nausea on the Likert scale (0-3), which was mainly used for assessing nausea in Japan (8,10-12), and adverse events according to the Common Terminology Criteria for Adverse Events, version 4.0 (Figure 1). The questionnaire regarding somnolence particularly asked about whether there was an impact on daily life.

\section{Statistical analysis}

Patients who received the scheduled carboplatin-based chemotherapy regimen were potentially eligible for the study, and patients who took olanzapine for breakthrough nausea and vomiting were analyzed as patients of the study. The primary endpoint was the total control [i.e., no emesis, no nausea (Likert scale 0 ), and no other rescue medications] rate for the overall period of $2-72 \mathrm{~h}$ after beginning olanzapine. Secondary endpoints were the evaluation of early efficacy using the improvement of the nausea score at 30,60 , and $120 \mathrm{~min}$ of taking olanzapine from baseline and adverse events.

In a previous trial, the rate of no nausea during $72 \mathrm{~h}$ after taking $10 \mathrm{mg}$ olanzapine for breakthrough nausea induced chemotherapy was $68 \%$ and metoclopramide was $23 \%$ (3). We expected that the antiemetic effect of $5 \mathrm{mg}$ of olanzapine would be equivalent to that of a 10-mg dose of the drug and that it would greater than the effect with metoclopramide. Therefore, a total control rate of $60 \%$ would indicate potential usefulness and $30 \%$ a lower limit of interest. A sample size of 22 patients was required to achieve an $\alpha$ level of 0.05 and statistical power of 0.90 . Considering that the incidence of nausea among patients receiving moderately 


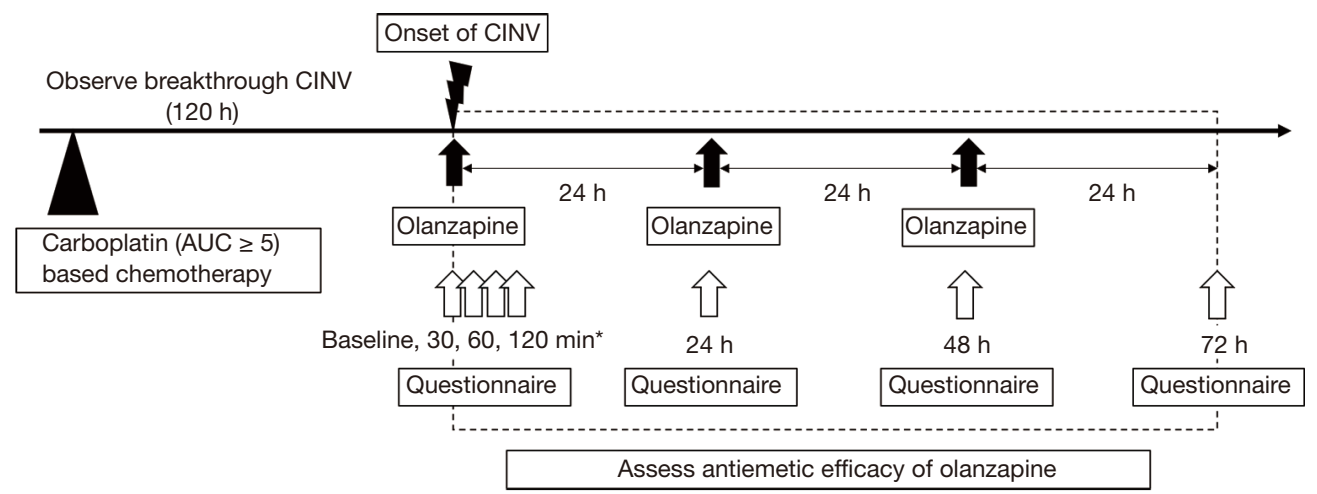

Figure 1 Monitoring diagram of the study. CINV, chemotherapy-induced nausea and vomiting. *A numerical scale questionnaire at baseline, 30,60 , and $120 \mathrm{~min}$.

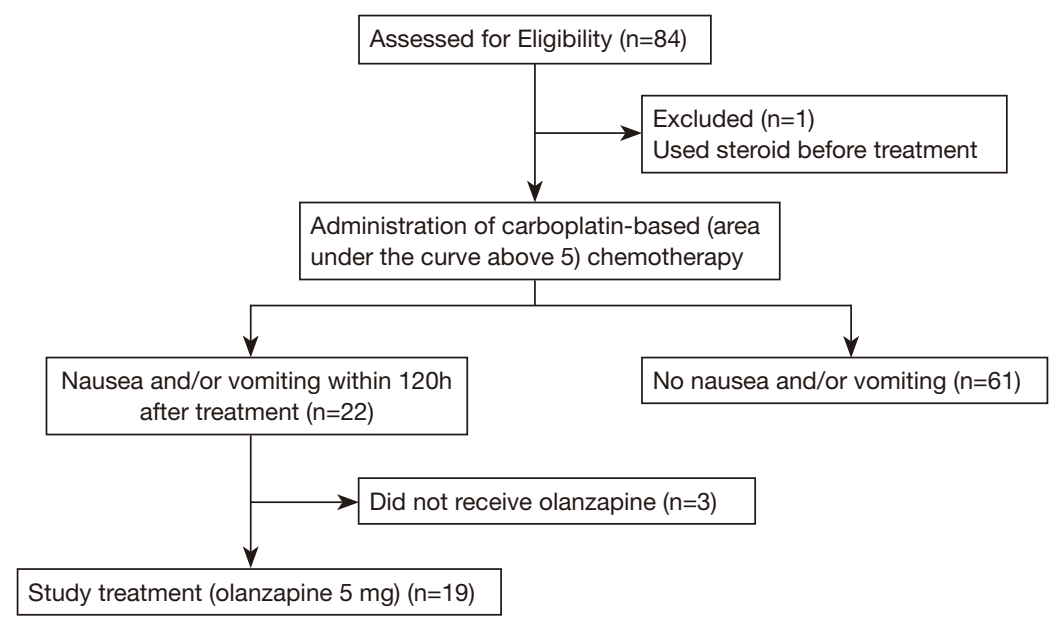

Figure 2 Consort diagram of the study.

emetic chemotherapy is reportedly about $10 \%$ to $40 \%$ (13), a maximum of 100 patients were thus needed for the study.

To evaluate the results of the nausea scale and the impact of each factor associated with somnolence, the Wilcoxon's signed rank and Fisher's exact tests were used. All calculations were performed using EZR version 1.38 (14).

\section{Results}

\section{Patient characteristics}

A total of 84 patients were potentially eligible for the study and received the scheduled carboplatin-based (area under the curve $\geq 5 \mathrm{mg} / \mathrm{mL} \cdot \min$ ) chemotherapy regimen. One patient was excluded because steroid treatment was started in the monitoring period. A total of 61 patients treated with chemotherapy had no nausea and vomiting. Moreover, three patients did not take olanzapine despite vomiting after chemotherapy. Remaining 19 (23\%) patients who took olanzapine for breakthrough nausea and vomiting were patients of the study (Figure 2). 15 (79\%) patients were inpatients and 4 (21\%) were outpatients.

The majority of patients had lung cancer $(n=16,84 \%$; Table 1), and no patient had poor renal function (serum creatinine $>2 \mathrm{mg} / \mathrm{dL}$ ). After carboplatin administration, the patients began taking olanzapine at a median of $69.8(8.3-118)$ h. Most patients (15, 79\%) started taking it in the daytime between 06:00 and 18:00.

\section{Efficacy}

The total control rate in patients who took olanzapine was 
Table 1 Patient characteristics

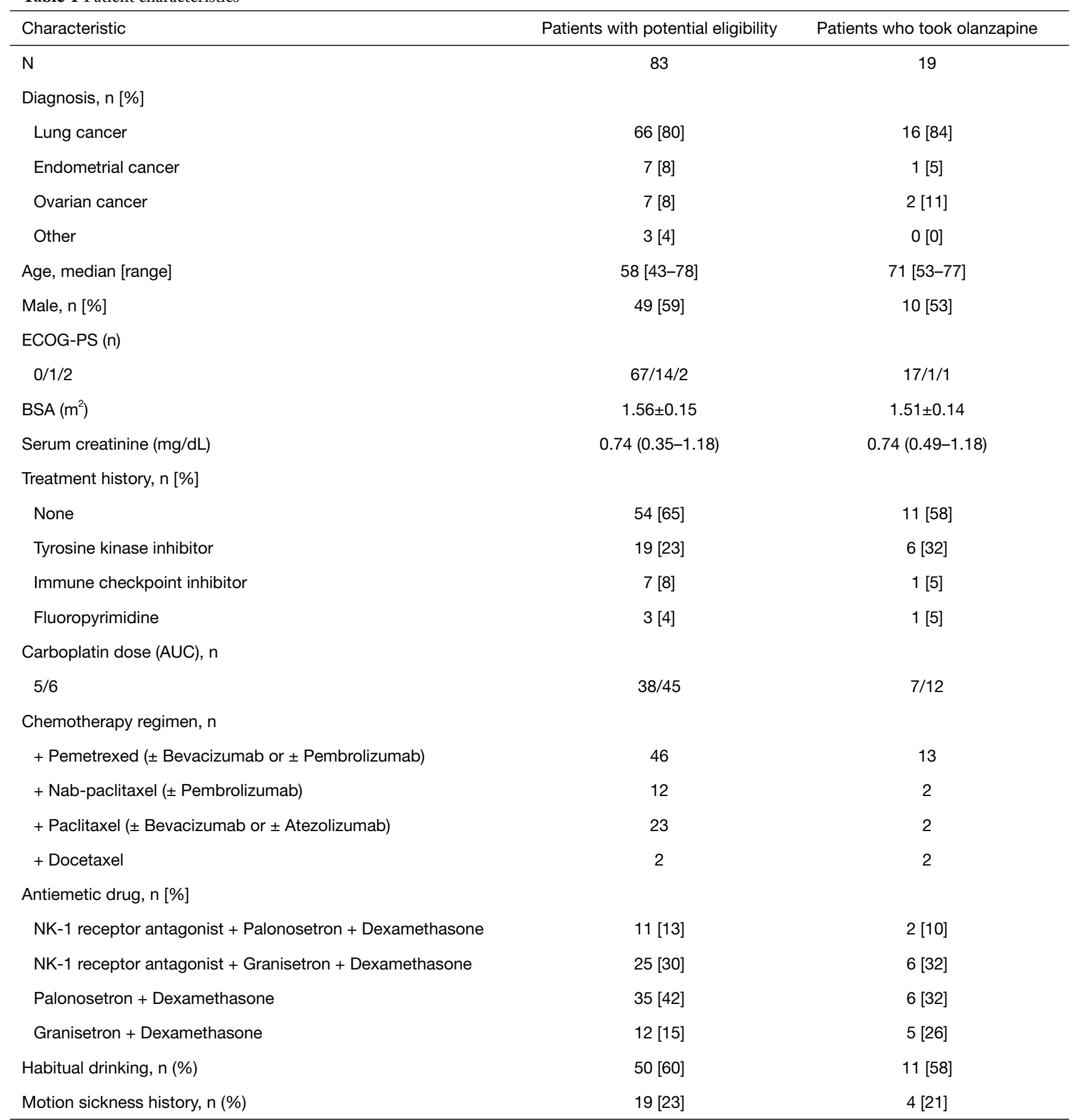

Table 1 (continued) 
Table 1 (continued)

\begin{tabular}{|c|c|c|}
\hline Characteristic & Patients with potential eligibility & Patients who took olanzapine \\
\hline \multicolumn{3}{|c|}{ Initiation period of olanzapine, $\mathrm{n}$ [\%] } \\
\hline $0-24 \mathrm{~h}$ & & $2[11]$ \\
\hline $24-48 \mathrm{~h}$ & & $2[11]$ \\
\hline $48-72 \mathrm{~h}$ & & $6[31]$ \\
\hline $96-120 \mathrm{~h}$ & & $3[16]$ \\
\hline
\end{tabular}

BSA, body surface area; ECOG-PS, Eastern Cooperative Oncology Group performance status; AUC, area under the curve; SD, standard deviation.

Table 2 Total control rate of nausea and vomiting with olanzapine

\begin{tabular}{|c|c|c|c|c|c|c|c|c|c|c|}
\hline Olanzapine outcomes & \multicolumn{3}{|c|}{$2-24 \mathrm{~h}(\mathrm{n}=19)$} & \multicolumn{3}{|c|}{$24-48$ h $(n=17)$} & \multicolumn{3}{|c|}{$48-72 \mathrm{~h}(\mathrm{n}=17)$} & $\begin{array}{l}\text { Overall }(2-72 h) \\
\quad(n=17)\end{array}$ \\
\hline \multicolumn{11}{|l|}{ Likert scale } \\
\hline 0 & $7[37]$ & 1 & 0 & $11[65]$ & 0 & 0 & 12 [70] & 0 & 1 & \\
\hline 2 & $1[5]$ & 1 & 0 & $2[12]$ & 1 & 0 & $2[12]$ & 1 & 0 & \\
\hline 3 & $0[0]$ & 0 & 0 & $0[0]$ & 0 & 0 & $0[0]$ & 0 & 0 & \\
\hline TC rate [\%] $(95 \% \mathrm{Cl})$ & & 32 [13-57] & & & 65 [38-89] & & & 65 [38-89] & & 29 [10-56] \\
\hline
\end{tabular}

Total control (TC) was defined as no episodes of emesis, nausea [Likert scale 0], or no use of rescue medications. Two patients were excluded from this analysis at $24-72 \mathrm{~h}$ and overall, because of discontinuation of olanzapine.

$32 \%$ [95\% confidence interval (CI): $13-57 \%$ ], $65 \%$ (95\% CI: $38-89 \%), 65 \%$ (95\% CI: $38-89 \%)$, and $29 \%$ (95\% CI: $10-56 \%$ ) during $2-24,24-48,48-72 \mathrm{~h}$, and overall period, respectively. The primary endpoint was thus not met because the total control rate during overall period did not exceed $60 \%$. In fact, it was less than the $30 \%$ lower limit of interest. In particular, it was markedly difficult to attain complete disappearance of nausea within $24 \mathrm{~h}$ after starting administration of olanzapine. However, total control rate $24 \mathrm{~h}$ onward was more than $60 \%$, as per our expectation (Table 2).

The numerical response scale at baseline, 30, 60, and 120 min were partially blank in two patients. In 17 patients who were all filled out, the median nausea score before beginning olanzapine was $3(1-8)$. The median decrease in the score was $3(0-5), 1(0-3)$, and $1(0-5)$ at 30, 60, and 120 min after taking olanzapine, respectively, with the score at 30 min being significantly decreased than before olanzapine administration $(\mathrm{P}=0.0078)$ (Figure 3). The maximal antiemetic effect was observed after $60 \mathrm{~min}$, at which point the score had been reduced by $67 \%$.

\section{Adverse events}

Somnolence of any grade was observed in $13(68 \%)$ patients, of whom $6(32 \%)$ had grade 2 and 1 (5\%) had grade 3 somnolence (Table 3). In all patients with grade 2 or higher, the somnolence occurred within $24 \mathrm{~h}$. Among the six patients with grade 2 somnolence, 3 took a reduced dose of $2.5 \mathrm{mg}$ beginning on day 2 and completed a 3 -day course of olanzapine. One patient chose to discontinue the olanzapine. The patient with grade 3 somnolence discontinued olanzapine because of a fall. One patient fainted but the attending physician diagnosed it as a vagal reflex. 


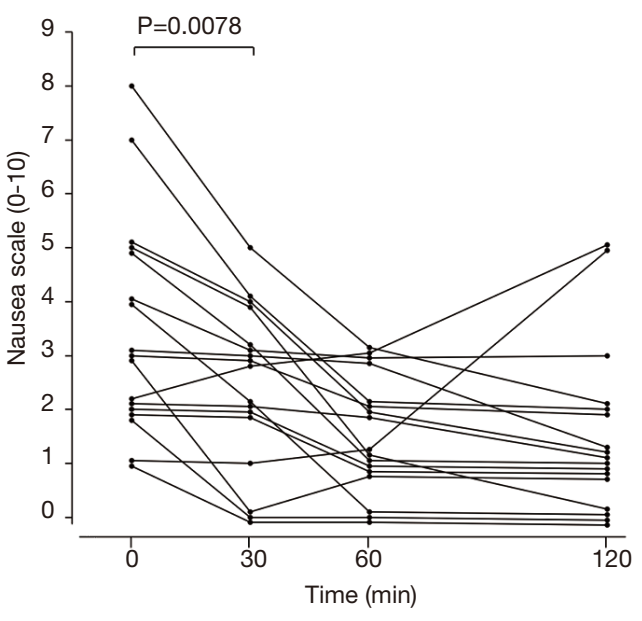

Figure 3 Nausea over time on a scale of $0-10$. The plot shows each patient's reported degree of nausea over time $(n=17)$. Statistical analysis was performed by Wilcoxon's signed rank test (time $0 \mathrm{vs}$. $30 \mathrm{~min})$.

\section{Discussion}

In the present study, $5 \mathrm{mg}$ of oral olanzapine did not exhibit the expected complete disappearance of the breakthrough CINV for the patients. The total control rate during $72 \mathrm{~h}$ after taking olanzapine was very low at $29 \%$; this could be attributed to no complete disappearance of nausea within the first $24 \mathrm{~h}$. However, the anticipated disappearance of nausea $24 \mathrm{~h}$ onward was noted on taking olanzapine every $24 \mathrm{~h}$.

In the study by Navari et al. (3), the rate of no nausea with a $10-\mathrm{mg}$ dose of olanzapine for breakthrough CINV after highly emetic chemotherapy (cisplatin, or doxorubicin and cyclophosphamide) was very high at $68 \%$. Our study evaluated the effects on breakthrough CINV after carboplatin-based chemotherapy, which could be lower risk of CINV than cisplatin, but the no nausea rate of $29 \%$ was far inferior to Navari's trial (3). This cause could be attributed to the difference in whether there was complete disappearance of nausea within the first $2 \mathrm{~h}$. The finding suggests that the antiemetic effect of a $5-\mathrm{mg}$ dose of olanzapine seems to be poorer compared with that of a 10-mg dose for breakthrough CINV regardless of the difference in chemotherapy, especially within the first 2-24 h after taking olanzapine. The cause might the maximum blood concentration of olanzapine takes 3-5 h after intake, so that the effective blood concentration was not immediately reached by 5 -mg dose.

However, in rescue treatment, it is important for the degree of nausea to decrease greatly if not completely disappear. Our results showed that the nausea scale score greatly reduced after $30 \mathrm{~min}$, and the score reduced by $67 \%$ (over two-thirds of baseline) after $60 \mathrm{~min}$, indicating that 5 -mg olanzapine was immediately effective. Considering that the disappearance of nausea $24 \mathrm{~h}$ onward could be expected by taking olanzapine every $24 \mathrm{~h}$, a dose of $5 \mathrm{mg}$ may be used as a rescue medication for breakthrough CINV if the 10-mg dose seems to be intolerant in patients.

The therapeutic effect may be influenced by patient characteristics and differences in preventive antiemetic therapy. Nausea control has been reported to be worse in women, those younger than 65 years, those who do not drink alcohol, those with a history of motion sickness, and those not using aprepitant or dexamethasone in antiemetic prophylaxis (15-17). Further, palonosetron exerts an antiemetic effect, which is better than that of granisetron in the delayed phase $(10,18)$. However, these nausea risk factors did not affect olanzapine's effect for breakthrough CINV.

Currently, the preventive antiemetic therapy of carboplatin (AUC $\geq 4 \mathrm{mg} / \mathrm{mL} \cdot \mathrm{min}$ ) is recommended in triple antiemetic therapy of aprepitant, 5HT3 receptor antagonist, and dexamethasone corresponds with highly emetogenic chemotherapy in NCCN guideline. However, at the start of this trial, triple antiemetic therapy with aprepitant was optional and unstandardized in Japan (19). Indeed, the patients with potential eligibility in our study, using palonosetron as the $5-\mathrm{HT}_{3}$ receptor antagonist, the incidence of breakthrough CINV was not also significantly different with or without aprepitant. Considering that $42 \%$ of eligible patients who received granisetron and dexamethasone without aprepitant as prophylactic antiemetic therapy had breakthrough CINV, it was again suggestive of the importance of the aprepitant combination for the selection of granisetron as a $5-\mathrm{HT}_{3}$ receptor antagonist for this prophylactic antiemetic therapy with carboplatin-based chemotherapy, which is based on the current guidelines. On the other hand, this study showed a reduction in the nausea scale score using olanzapine in almost all patients. Therefore, olanzapine at a dose of $5 \mathrm{mg}$ is expected to reduce the nausea score, even under various patient backgrounds and preventive antiemetic therapy.

Although the common side effects of olanzapine include hyperglycemia, these have been reported to be mostly tolerable in large-scale clinical trials $(6,8,20)$. Therefore, we did not evaluate blood glucose levels in the present study. A small number of patients reported constipation and malaise on the questionnaire, but in all cases, the symptoms were mild and the attending physicians thought that they were 
Table 3 Adverse events with olanzapine ( $n=19)$

\begin{tabular}{|c|c|c|c|c|}
\hline Adverse events & Grade 1 & Grade 2 & Grade 3 & All Grades \\
\hline Constipation & $2(11)$ & $0(0)$ & $0(0)$ & $2(11)$ \\
\hline Sweating & $1(5)$ & $0(0)$ & $0(0)$ & $1(5)$ \\
\hline Malaise & $1(5)$ & $0(0)$ & $0(0)$ & $1(5)$ \\
\hline Fall & $0(0)$ & $1(5)$ & $0(0)$ & $1(5)$ \\
\hline
\end{tabular}

Data are presented as $\mathrm{n}(\%)$.

not very likely to have been caused by olanzapine.

Most patients in this study took olanzapine during the daytime. Previous clinical studies have reported that with administration of olanzapine in the morning, dizziness and somnolence are tolerable $(4,21)$. However, 7 (37\%) patients had grade 2 or 3 somnolence within 24 h, and $2(22 \%)$ discontinued the drug. In particular, one patient had grade 3 somnolence and fell after taking olanzapine. It is unclear whether these were olanzapine-related adverse event, since this study did not have a placebo group. However, if it is prescribed, care must be taken with regard to the possibility of somnolence, with adequate monitoring for somnolence being available for $24 \mathrm{~h}$ after administration.

Olanzapine-induced somnolence was reported to be acceptable in a large-scale clinical trial of patients (6). However, most such information is based on trials of prophylactic antiemetic therapy, and a previous retrospective study has reported results similar to ours, stating that $13 \%$ of patients discontinued the olanzapine rescue due to sedation (9), so further research is needed on the tolerability of olanzapine for breakthrough CINV.

The chief limitation of the present study was its single-arm nature and small size. As mentioned, with the development of prophylaxis antiemetic therapy, if trying to conduct a multigroup comparative study, the enrolment of subjects with onset of breakthrough CINV requires an enormous number of patient registrations. Since the evidence of $5 \mathrm{mg}$ olanzapine for breakthrough CINV was little, we conducted a preliminary trial with a small number of patients as a step to be a comparative study. This is based on ethical considerations to reduce the number of patients who receive unnecessary intervention.

We could not achieve the planned number of patients ( $n=22$ ) who needed olanzapine for breakthrough symptoms. The incidence of breakthrough CINV in enrolled patients was within expectations; however, adequate planned number of patients could not be registered during the study period. One of the causes for the low enrolment might be that the number of patients that met the diabetic history exclusion criteria exceeded our expectations. However, the statistical power based on the included 19 patients was calculated to be $87.1 \%$, and we consider this to be an adequate number of patients to investigate the study question. Hence, the present study can be validated as a preliminary study to evaluate the efficacy of a 5-mg dose of olanzapine for breakthrough CINV.

5 -mg dose of olanzapine was not efficacious as expected. While there are few verification studies about the optimal dose of olanzapine for breakthrough CINV, it is an issue that should be continued for consideration using various study designs.

\section{Conclusions}

Our findings suggest that a 5-mg dose of olanzapine is less effective for the complete disappearance of breakthrough CINV, there seems to be no superior overall effect compared with historic data obtained by the administration of $10 \mathrm{mg}$ olanzapine, especially within $24 \mathrm{~h}$.

\section{Acknowledgments}

We thank the patients for their participation in this study, the study management team and staff at individual study sites, and particularly, the following investigators: Toyoaki Hida and Masaki Kajita (Aichi Cancer Center Hospital); Takashi Shibata, Osamu Yamamuro, and Tomohiko Ogasawara (Japanese Red Cross Nagoya Daini Hospital); Chiyoe Kitagawa, Hideo Saka, and Masahiko Nakai (National Hospital Organization Nagoya Medical Center); Akihito Kubo and Sumiyo Tsukiyama (Aichi Medical University); 
Masaki Matsuo and Shogo Arakawa (Chubu Rosai Hospital); Fumio Nomura (Japanese Red Cross Nagoya Daiichi Hospital); Shigehiko Morikawa and Yasuhiro Toda (Komaki City Hospital); Kazuyoshi Imaizumi, Yasuhiro Goto, and Shigeki Yamada (Fujita Health University School of Medicine); Kenji Akita and Yasuo Nakamura (Nagoya City West Medical Center); Ken Maeno and Kazunori Kimura (Nagoya City University Hospital).

Funding: None.

\section{Footnote}

Reporting Checklist: The authors have completed the TREND reporting checklist. Available at http://dx.doi. org/10.21037/apm-20-1784

Data Sharing Statement: Available at http://dx.doi. org/10.21037/apm-20-1784

Conflicts of Interest: All authors have completed the ICMJE uniform disclosure form (available at http://dx.doi. org/10.21037/apm-20-1784). The authors have no conflicts of interest to declare.

Ethical Statement: The authors are accountable for all aspects of the work in ensuring that questions related to the accuracy or integrity of any part of the work are appropriately investigated and resolved. This clinical trial was approved by the Ethics Committee of each hospital (No.: 2018-1-282) and was conducted in accordance with the Declaration of Helsinki (as revised in 2013). This study was registered with the University Hospital Medical Information Network Clinical Trials Registry (UMINCTR) (Study ID: UMIN000031823).

Open Access Statement: This is an Open Access article distributed in accordance with the Creative Commons Attribution-NonCommercial-NoDerivs 4.0 International License (CC BY-NC-ND 4.0), which permits the noncommercial replication and distribution of the article with the strict proviso that no changes or edits are made and the original work is properly cited (including links to both the formal publication through the relevant DOI and the license). See: https://creativecommons.org/licenses/by-nc-nd/4.0/.

\section{References}

1. Glare P, Pereira G, Kristjanson LJ, et al. Systematic review of the efficacy of antiemetics in the treatment of nausea in patients with far-advanced cancer. Support Care Cancer 2004;12:432-40.

2. Jones JM, Qin R, Bardia A, et al. Antiemetics for chemotherapy-induced nausea and vomiting occurring despite prophylactic antiemetic therapy. J Palliat Med 2011;14:810-4.

3. Navari RM, Nagy CK, Gray SE. The use of olanzapine versus metoclopramide for the treatment of breakthrough chemotherapy-induced nausea and vomiting in patients receiving highly emetogenic chemotherapy. Support Care Cancer 2013;21:1655-63.

4. Chanthawong S, Subongkot S, Sookprasert A. Effectiveness of olanzapine for the treatment of breakthrough chemotherapy induced nausea and vomiting. J Med Assoc Thai 2014;97:349-55.

5. Einhorn LH, Rapoport B, Navari RM, et al. 2016 updated MASCC/ESMO consensus recommendations: prevention of nausea and vomiting following multiple-day chemotherapy, high-dose chemotherapy, and breakthrough nausea and vomiting. Support Care Cancer 2017;25:303-8.

6. Navari RM, Qin R, Ruddy KJ, et al. Olanzapine for the Prevention of Chemotherapy-Induced Nausea and Vomiting. N Engl J Med 2016;375:134-42.

7. Miya T, Kobayashi K, Hino M, et al. Efficacy of triple antiemetic therapy (palonosetron, dexamethasone, aprepitant) for chemotherapy-induced nausea and vomiting in patients receiving carboplatin-based, moderately emetogenic chemotherapy. Springerplus 2016;5:2080.

8. Yanai T, Iwasa S, Hashimoto H, et al. A double-blind randomized phase II dose-finding study of olanzapine $10 \mathrm{mg}$ or $5 \mathrm{mg}$ for the prophylaxis of emesis induced by highly emetogenic cisplatin-based chemotherapy. Int J Clin Oncol 2018;23:382-8.

9. Chiu L, Chiu N, Chow R, et al. Olanzapine for the prophylaxis and rescue of chemotherapy-induced nausea and vomiting (CINV): a retrospective study. Ann Palliat Med 2016;5:172-8.

10. Suzuki K, Yamanaka T, Hashimoto H, et al. Randomized, double-blind, phase III trial of palonosetron versus granisetron in the triplet regimen for preventing chemotherapy-induced nausea and vomiting after highly emetogenic chemotherapy: TRIPLE study. Ann Oncol 2016;27:1601-6.

11. Hashimoto H, Abe M, Tokuyama O, et al. Olanzapine 5 $\mathrm{mg}$ plus standard antiemetic therapy for the prevention of chemotherapy-induced nausea and vomiting (J-FORCE): a multicentre, randomised, double-blind, placebo- 
controlled, phase 3 trial. Lancet Oncol 2020;21:242-9.

12. Nishimura J, Satoh T, Fukunaga $M$, et al. Combination antiemetic therapy with aprepitant/fosaprepitant in patients with colorectal cancer receiving oxaliplatin-based chemotherapy (SENRI trial): a multicentre, randomised, controlled phase 3 trial. Eur J Cancer 2015;51:1274-82.

13. Jordan $\mathrm{K}$, Blattermann L, Hinke A, et al. Is the addition of a neurokinin-1 receptor antagonist beneficial in moderately emetogenic chemotherapy?-a systematic review and metaanalysis. Support Care Cancer 2018;26:21-32.

14. Kanda Y. Investigation of the freely available easy-touse software 'EZR' for medical statistics. Bone Marrow Transplant 2013;48:452-8.

15. Tamura K, Aiba K, Saeki T, et al. Testing the effectiveness of antiemetic guidelines: results of a prospective registry by the CINV Study Group of Japan. Int J Clin Oncol 2015;20:855-65.

16. Dranitsaris G, Molassiotis A, Clemons M, et al. The development of a prediction tool to identify cancer patients at high risk for chemotherapy-induced nausea and vomiting. Ann Oncol 2017;28:1260-7.

17. Grunberg SM. Antiemetic activity of corticosteroids in

Cite this article as: Maeda A, Yoshida H, Inoue H, Ejiri M, Yamaguchi S, Kushihara H, Yamamoto Y, Ando Y, Sato Y, Tashiro Y, Hasegawa A, Takahara Y, Mizutani M, Oze I, Shimizu J. Effects of 5-mg dose of olanzapine for breakthrough nausea and vomiting in patients receiving carboplatinbased chemotherapy: a prospective trial. Ann Palliat Med 2021;10(3):2699-2708. doi: 10.21037/apm-20-1784 patients receiving cancer chemotherapy: dosing, efficacy, and tolerability analysis. Ann Oncol 2007;18:233-40.

18. Saito M, Aogi K, Sekine I, et al. Palonosetron plus dexamethasone versus granisetron plus dexamethasone for prevention of nausea and vomiting during chemotherapy: a double-blind, double-dummy, randomised, comparative phase III trial. Lancet Oncol 2009;10:115-24.

19. Kusagaya H, Inui N, Karayama M, et al. Evaluation of palonosetron and dexamethasone with or without aprepitant to prevent carboplatin-induced nausea and vomiting in patients with advanced non-small-cell lung cancer. Lung Cancer 2015;90:410-6.

20. Navari RM, Gray SE, Kerr AC. Olanzapine versus aprepitant for the prevention of chemotherapy-induced nausea and vomiting: a randomized phase III trial. J Support Oncol 2011;9:188-95.

21. Abe M, Hirashima Y, Kasamatsu Y, et al. Efficacy and safety of olanzapine combined with aprepitant, palonosetron, and dexamethasone for preventing nausea and vomiting induced by cisplatin-based chemotherapy in gynecological cancer: KCOG-G1301 phase II trial. Support Care Cancer 2016;24:675-82. 